JPE 10-3-4

\title{
Digital Control Strategy for Input-Series-Output-Parallel Modular DC/DC Converters
}

\author{
Deshang Sha ${ }^{\dagger}$, Zhiqiang Guo*, and Xiaozhong Liao* \\ $\dagger *$ School of Automation, Beijing Institute of Technology, China
}

\begin{abstract}
Input-series-output-parallel (ISOP) converters consisting of multiple modular DC/DC converters can enable low voltage rating switches to be used under high voltage input applications. This paper presents a digital control strategy, which can achieve equal sharing of input voltage for a modular ISOP system consisting of two-transistor forward DC/DC converters by forcing the input voltages of neighboring modules to be equal. The proposed scheme is analyzed using small signals analysis based on the state space average method. The performance of the proposed control strategy is verified with an experimental prototype of an ISOP converter made up of three two-switch forward converters.
\end{abstract}

Key Words: Digital control, Forward DC/DC, Input-series and output-parallel, Modular DC/DC converters, Voltage sharing

\section{INTRODUCTION}

Multilevel converters enable low-voltage rating switches to be used under high voltage applications. However, system reliability cannot be guaranteed for a large quantity of diodes or flying capacitors [1]. The input-series output-parallel (ISOP) configuration consists of several modular DC-DC converters connected in series at the input and in parallel at the output. This enables the use of high switching frequency metal oxide semiconductor field effect transistors (MOSFETs) with low voltage ratings, which leads to high power density and high conversion efficiency. However, control must be taken to ensure equal input voltage sharing among all of the modules for the ISOP DC/DC converters. Several control schemes have been proposed to achieve this purpose. Common duty ratio control results in stable operation for ISOP converters [2], but equal input voltage sharing (IVS) can not be achieved for parameter mismatches such as a turns ratio mismatch of transformers. A charge control scheme with input-voltage feed forward has been implemented for a two-converter system [3]. However, the input currents have to been sensed as well as the input voltages. A master/slave control schemes can only be used in small applications due to electrical isolation problems [4]. A three-loop control scheme [5], [6] and a decoupling IVS control scheme [7] can achieve input voltage equally for an ISOP system. However, all of the voltage references for the IVS control loops must vary with the total input voltage, thus complicating the design. Although interleaving

Manuscript received Dec. 16, 2009; revised Apr. 10, 2010

$\dagger$ Corresponding Author: shadeshang@ @it.edu.cn

Tel: +86-10-68912460, Beijing Institute of Technology

* School of Automation, Beijing Institute of Technology, China control can minimize the output current ripples, in order to achieve satisfactory control, the integrated circuits need to be synchronized [4], [5], which complicates the design further. Most ISOP connected modules under research are full-bridge or single-ended forward DC-DC converters [2]-[10]. It is well known that neither of these are well suited because fullbridge converters work at the risk of shoot-through failures for switching devices connected directly across the source. Additional demagnetizing or snubber circuits have to be designed for single-ended forward converters. However, two-transistor forward (TTF) DC/DC converters are well suited for use under high input voltage and high power applications due to their inherent advantages. These advantages include automatic voltage spike clamping and demagnetization through diodes connected to the switching devices. Dual TTF DC/DC converters with all of the windings sharing one transformer can be fed from input DC voltage, but each of them are coupled through a magnetizing field and as a result they can not been called truly modular converters from an architecture point of view [11]. Control implementation for ISOP DC/DC converters using analogy circuits is time consuming and inflexible [2]-[11]. With the emergence of digital signal processors (DSP), digital control is becoming a potentially attractive alternative to the analogy option due to its inherent advantages [12], [13].

This paper proposes a very simple digital IVS control for ISOP systems, which does not require changing the voltage references for each module. Perfect voltage sharing can be achieved by forcing the input voltage between the neighbor modules equally, no matter what the total input voltage is. The proposed ISOP configuration consists of two-switch forward DC-DC converters, which have the advantages of no risks 


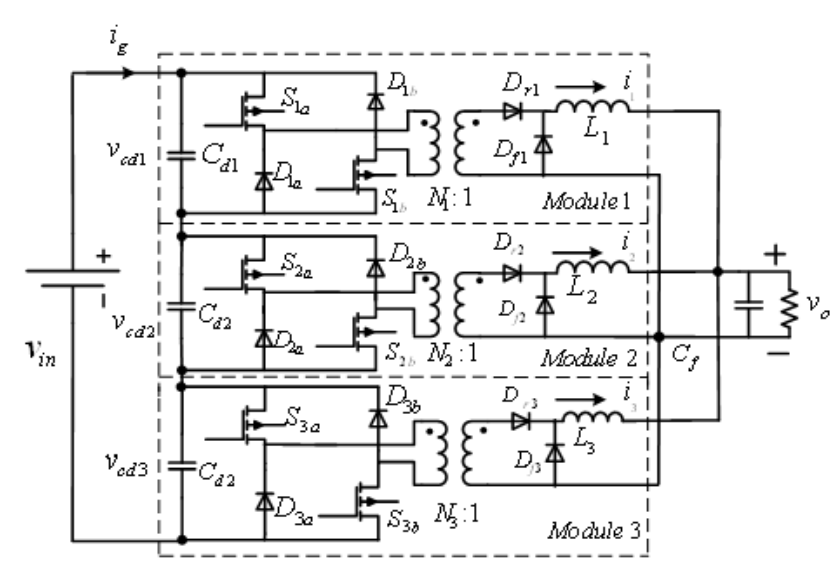

Fig. 1. ISOP connected modular two-switch forward converters.

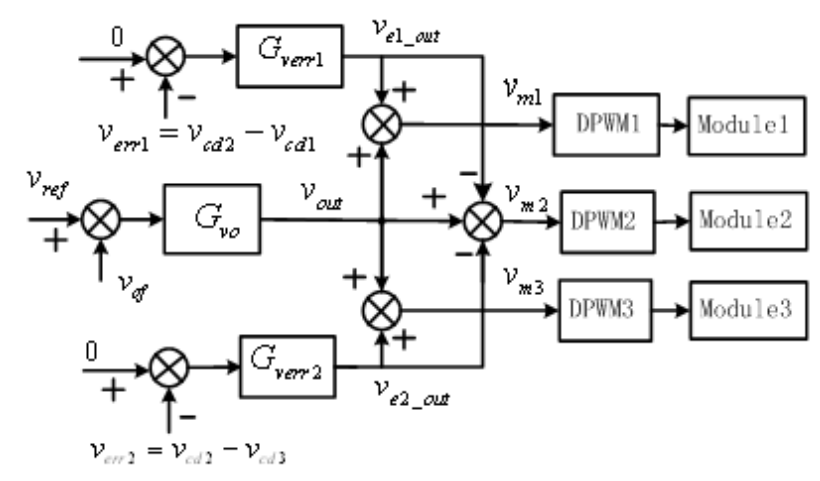

Fig. 2. Proposed control strategy.

of shooting through, automatic voltage spike clamping and demagnetizing through diodes. Loop gain analysis is carried out on the proposed control strategy and a $100 \mathrm{~W}$ prototype is fabricated and experimentally evaluated.

This paper is organized as follows. The proposed digital control strategy is presented and analyze in Section II. Small signal analysis and loop gain analysis are provided in Section III. Experimental results of the prototype are illustrated in Section IV. Conclusions are given in Section V.

\section{Novel Input Voltage Sharing Control}

\section{A. Operation principles of the proposed control strategy}

Fig. 1 shows the schematic of an ISOP converter made up of three TTF DC/DC converters. In this configuration, the total input voltage $v_{i n}$ is divided by the input capacitors $C_{d 1}, C_{d 2}$ and $C_{d 3}$ thus we get the voltages $v_{c d 1}, v_{c d 2}$ and $v_{c d 3}$ working as individual input voltages for each module respectively.

Equal sharing of the total input voltage $v_{i n}$ means that $v_{c d 1}=v_{c d 2}=v_{c d 3}$ which can also be described by:

$$
\left\{\begin{array}{l}
v_{c d 1}-v_{c d 2}=0 \\
v_{c d 2}-v_{c d 3}=0
\end{array}\right.
$$

However, satisfying (1) is only one of our control objectives. Another is that the output voltage should stay unchanged during load and under input voltage variations. Hence, the control strategy consists of one common output voltage regulation (OVR) loop and two IVS regulation loops. Fig. 2

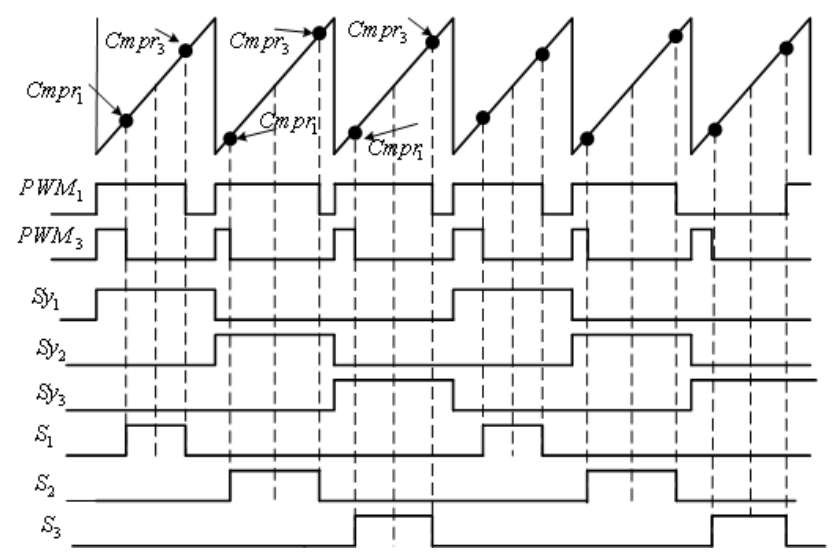

Fig. 3. Schematic of digital PWM generation.

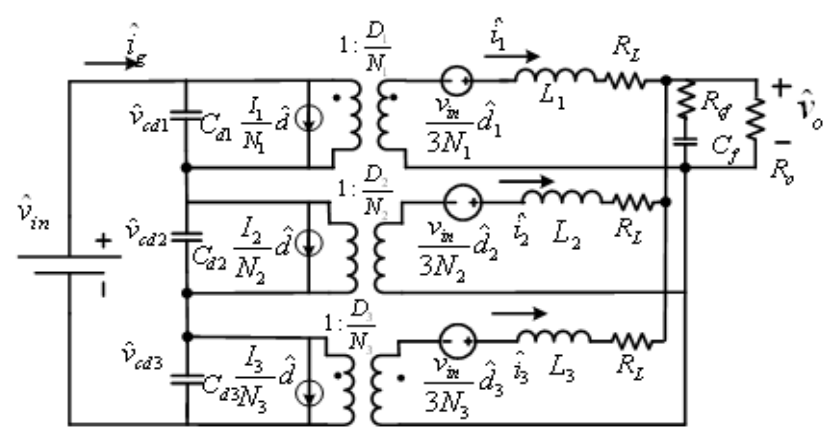

Fig. 4. Small-signal average model of the converter.

shows a control block which can achieve equal sharing of the input voltage and a constant voltage output, where $v_{r e f}$ is the reference voltage for the voltage output; $v_{o f}$ is the feedback of the output voltage; $G_{v o}$ is the compensation network of the output voltage control. To satisfy (1), both of the differences between the input voltages of neighboring modules must be equal to zero. This is why the references are zero for both of the input voltage sharing control loops. Input voltage differences are amplified by the compensation networks $G_{v e r r 1}$ and $G_{v e r r 2}$. Then we can get $c$ and $v_{e 2 \_o u t}$, which work as offset voltages during duty ratio generation. $v_{\text {out }}$ works as the output of OVR. Through linear combinations of OVR and IVS outputs, the values determining the duty ratios of individual modules can be derived as shown in (2).

$$
\left\{\begin{array}{l}
v_{m 1}=v_{\text {out }}+v_{\text {e1_out }} \\
v_{m 2}=v_{\text {out }}-v_{m 1}-v_{m 3} \\
v_{m 3}=v_{\text {out }}+v_{\text {e2_out }} .
\end{array}\right.
$$

The balancing mechanism can be seen in Fig. 2 and can be explained as follows, if $v_{c d 2}$ is equal to $v_{c d 3}$ and both of them are higher than $v_{c d 1}$, then $v_{e r r 1}$ is positive and the calculation of $G_{\text {verr } 1}$ causes $v_{\text {e1_out }}$ to decrease. This results in a decrease in the duty ratio for module 1 and an increase in the duty ratio for module 2 . Because $v_{e r r}$ is equal to zero in such case, $v_{e 2 \_o u t}$ remains unchanged. Therefore, capacitor $C_{d 1}$ is charged while capacitor $C_{d 2}$ is discharged, leading to an increase in $v_{c d 1}$ and a decrease in $v_{c d 2}$. On the other hand, when $v_{c d 2}$ is equal to $v_{c d 1}$ and both of them are higher than $v_{c d 3}$, through the calculation with the proposed control 


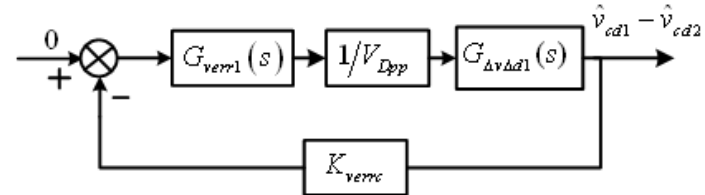

(a)

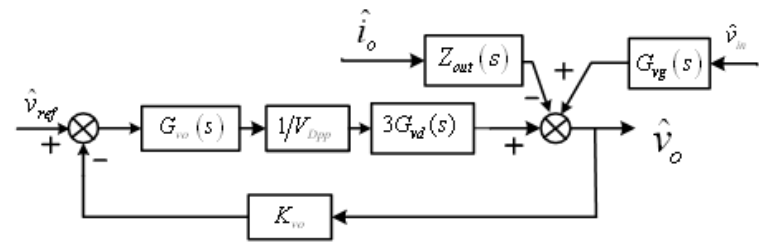

(b)

Fig. 5. System block diagram of ISOP converter (a) IVS control block diagram; (b) OVR control block diagram.

strategy, $v_{c d 3}$ will increase while $v_{c d 2}$ decreases. In this way, all of the individual input voltages become equal and power sharing balance among all of the modular DC/DC converters is accomplished.

\section{B. Digital implementation of the control strategy}

Note that the implementation of the proposed control strategy, shown in Fig. 2, is implemented by only one digital signal processor (DSP). Compensation networks including both OVR and IVS are based on digital proportion-integral types. The gate signals for the switches of each modular DC/DC converter are interleaved.

Fig. 3 shows the interleaved gate signal generation. Timer 1 is put in continuous mode and its period register is loaded with a value corresponding to the desired switching frequency and the number of modules. Because there are three modules, the frequency of Time 1 is three times that of the switching frequency for each module. Gate signals for every module are generated every three periods of Timer 1. Using module 1 as an example, the value of the compare register is constantly compared with the value of the time counter. When the values match, a transition from high to low takes place on the associated outputs form $P W M 1$ and $P W M 3$. The compared values can be seen from (3):

$$
\left\{\begin{array}{l}
C m p r_{1}=T_{P 1} / 2-v_{m 1} / 2 \\
C m p r_{3}=T_{P 1} / 2+v_{m 1} / 2
\end{array}\right.
$$

where Tpl is the value of the period register. For module 2 and module 3 , the signals $P W M 1$ and $P W M 3$ are generated in the same way except that $v_{m 2}$ and $v_{m 3}$ supersede $v_{m 1}$ respectively. Each period of Time 1 is marked subsequently by the synchronous signals $S y_{1}, S y_{2}$ and $S y_{3}$ respectively. The logic expression of the gate signals for all of the switches in the ISOP configuration is shown in (4):

$$
\left\{\begin{array}{l}
S_{1}=\left(P W M_{1} \oplus P W M_{3}\right) \cdot S y_{1} \\
S_{2}=\left(P W M_{1} \oplus P W M_{3}\right) \cdot S y_{2} \\
S_{3}=\left(P W M_{1} \oplus P W M_{3}\right) \cdot S y_{3}
\end{array}\right.
$$

where $S_{1}, S_{2}$ and $S_{3}$ are the gate signals for the switches of module 1 , module 2 and module 3 respectively.

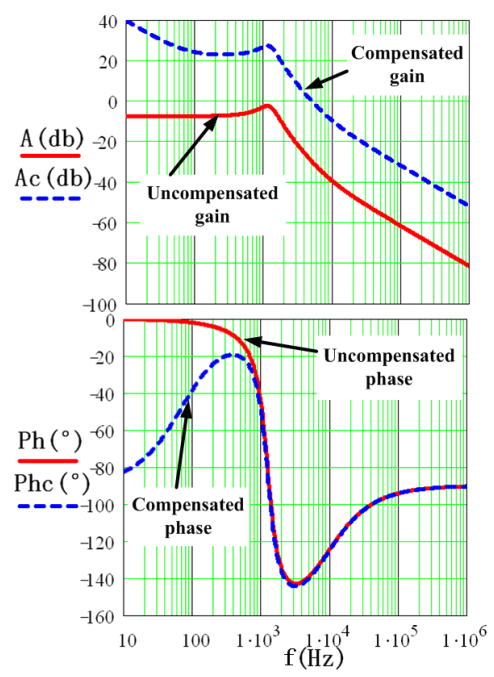

Fig. 6. Output voltage regulation (OVR) loop gains.

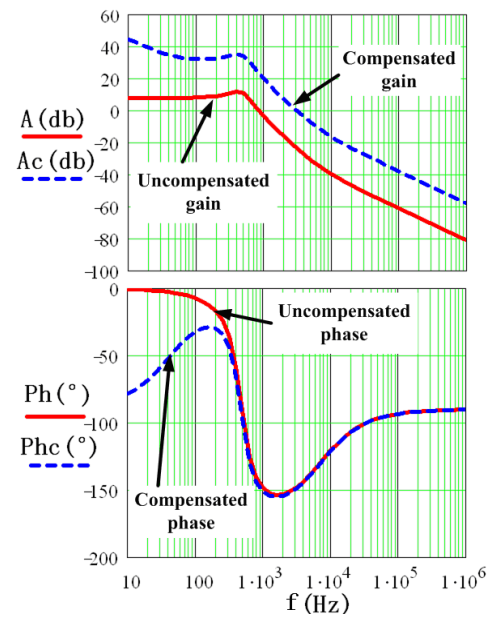

Fig. 7. Input voltage sharing (IVS) control loop gains.

\section{Small Signal AnAlysis AND Regular LoOP GAIN DESIGN}

\section{A. Small signal modeling of the ISOP system}

Based on a small signal model of isolated buck DC-DC converters, we can derive the small signal model of a ISOP connected three-converter system, as shown in Fig. 4, where $\hat{d}$ is the perturbation of the duty ratio, while $\hat{v}_{c d i}(i=1,2,3)$ and $\hat{i}_{i}(i=1,2,3)$ represent perturbations of the individual input voltages and output currents. Equivalent series resistors of the output inductors and output capacitors are denoted as $R_{L}$ and $R_{c f}$ respectively. For simplicity of the analysis, the three converters are assumed to have the same turns ratio $N$ and same output inductance $L$, which can be revealed from (5):

$$
\left\{\begin{array}{l}
N_{1}: 1=N_{2}: 1=N_{3}: 1=N: 1 \\
L_{1}=L_{2}=L_{3}=L
\end{array} .\right.
$$

Under the steady state, the three converters share the input voltage and load current equally. This can be seen as follows:

$$
V_{o}=V_{c d 1} \frac{D_{1}}{n}=V_{c d 2} \frac{D_{2}}{n}=V_{c d 3} \frac{D_{3}}{n} .
$$




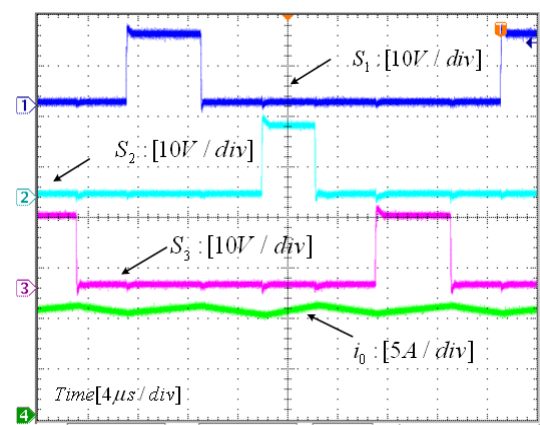

Fig. 8. Gate signals for each module switches.

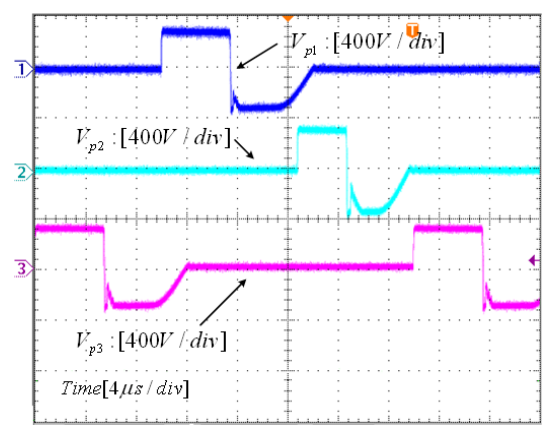

Fig. 9. Primary voltages over individual transformers.

Because we assume the duty ratios of all the modules are the same:

$$
D_{1}=D_{2}=D_{3}=D \text {. }
$$

Thus, we can get:

$$
\left\{\begin{array}{c}
I_{1}=I_{2}=I_{3}=\frac{I_{0}}{3}=\frac{V_{0}}{3 R_{0}} \\
V_{c d 1}=V_{c d 2}=V_{c d 3}=\frac{V_{i n}}{3}
\end{array}\right.
$$

where $I_{1}, I_{2}$ and $I_{3}$ are the individual steady-state output currents, $R_{0}$ is the rated load and $V_{i n}$ is the value of the total input voltage at the steady state. $I_{0}$ and $V_{0}$ are the load current and output voltage under the steady states. According to Fig. 4 and (5), the small model equations can be expressed as:

$$
\begin{aligned}
& \left\{\begin{array}{l}
\frac{D_{1}}{N} \hat{v}_{c d 1}+\frac{v_{i n}}{3 N} \hat{d}_{1}=\left(s L+R_{L}\right) \cdot \hat{i}_{1}+\hat{v}_{o} \\
\frac{D_{2}}{N} \hat{v}_{c d 2}+\frac{v_{i n}}{3 N} \hat{d}_{2}=\left(s L+R_{L}\right) \cdot \hat{i}_{2}+\hat{v}_{o} \\
\frac{D_{3}}{n} \hat{v}_{c d 3}+\frac{v_{i n}}{3 N} \hat{d}_{3}=\left(s L+R_{L}\right) \cdot \hat{i}_{3}+\hat{v}_{o}
\end{array}\right. \\
& \left\{\begin{array}{l}
\hat{i}_{g}-\hat{v}_{c d 1} \cdot s C_{d}-\frac{i_{1}}{N} \hat{d}_{1}=\frac{D_{1}}{N} \cdot \hat{i}_{1} \\
\hat{i}_{g}-\hat{v}_{c d 2} \cdot s C_{d}-\frac{i_{2}}{N} \hat{d}_{2}=\frac{D_{2}}{N} \cdot \hat{i} \\
\hat{i}_{g}-\hat{v}_{c d 3} \cdot s C_{d}-\frac{i_{3}}{N} \hat{d}_{3}=\frac{D_{3}}{N} \cdot \hat{i}
\end{array}\right.
\end{aligned}
$$

From Fig. 4 we can also get:

$$
\left\{\begin{array}{l}
\hat{v}_{i n}=\hat{v}_{c d 1}+\hat{v}_{c d 2}+\hat{v}_{c d 3} \\
\hat{i}_{o}=\hat{i}_{1}+\hat{i}_{2}+\hat{i}_{3}=\hat{v}_{o}\left[R_{o} / /\left(R C_{f}+\frac{1}{S C_{f}}\right)\right] .
\end{array}\right.
$$

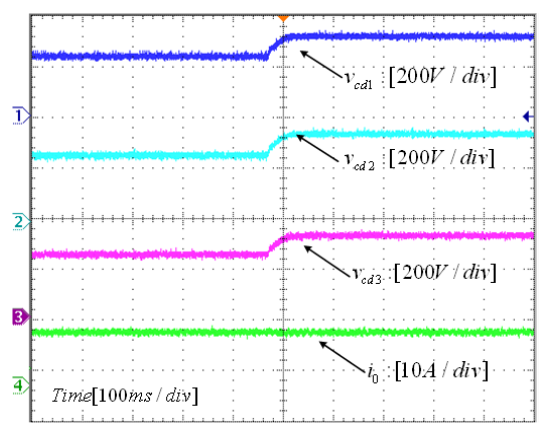

Fig. 10. Response to a step change of the input voltage $V_{i n}$.

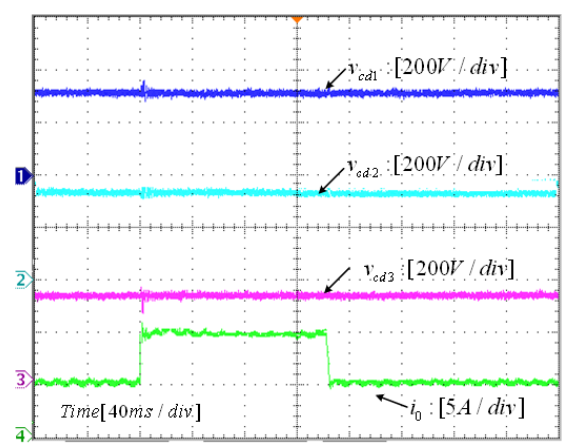

Fig. 11. Response to a step change of the load.

Based on (9), (10) and (11), by setting $\hat{v}_{i n}=0$ and $\hat{d}_{k}=0$, where $k \neq i$, the control-to-output transfer function of an arbitrary module $i(i=1,2,3)$ can be written as:

$$
\begin{aligned}
& G_{v d i}=\left.\frac{\hat{v}_{o}}{\hat{d}_{i}}\right|_{\hat{v}_{i n}=0} \\
& \hat{d} k=0(k \neq i) \\
& =\frac{\frac{v_{i n}}{3 N}\left(s C_{f} R_{o}+1\right)}{s^{2} L C_{f}\left(1+\frac{R_{c f}}{R_{o}}\right)+s\left[\frac{L}{R_{o}}+R_{L} C_{f}\left(1+\frac{R_{c f}}{R_{o}}\right)+3 C_{f} R_{c f}\right]+\frac{R_{L}}{R_{o}}+3} .
\end{aligned}
$$

The control-to-module-input transfer function can be shown as follows:

$$
\begin{gathered}
G_{\Delta v \Delta d_{i}}=\left.\frac{\hat{v}_{c d 1}-\hat{v}_{c d(i+1)}}{\hat{d}_{i}-\hat{d}_{i+1}}\right|_{\begin{array}{c}
\hat{v}_{i n}=0 \\
\hat{d} k=0(k \neq 1)
\end{array}} \\
=-\frac{s \frac{V_{o} L_{f}}{3 n R_{o}}+\frac{V_{o} R_{L}}{3 n R_{o}}+\frac{V_{i n} D}{3 n^{2}}}{s^{2} C_{d} L_{f}+s C_{d} R_{L}+\left(\frac{D}{n}\right)^{2}} .
\end{gathered}
$$

\section{B. Regulator Loop Design}

The specifications of the ISOP system are shown in Table I:

There is one OVR control loop and two IVS loops sharing the same structure and parameters with the proposed control scheme. Thus, we can take one IVS control loop gains as an example. Fig. 5 shows a system block diagram, where $G_{v e r r 1}(s)$ and $G_{v 0}(s)$ are the transfer functions of the compensators for one IVS and one OVR respectively. $V_{D p p}$ is the amplitude of a digital triangle waveform although it is a 


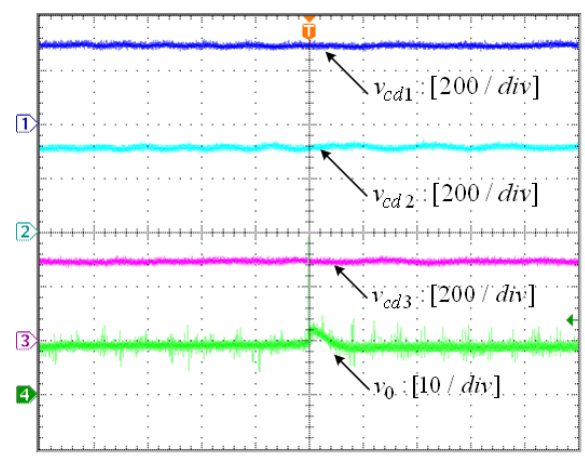

Fig. 12. Response to the load stepping down.

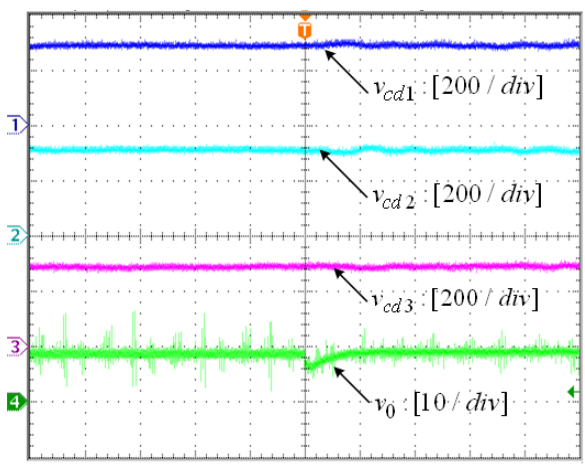

Fig. 13. Response to the load stepping up.

dummy, which can be calculated through the A/D sampling results and the values of the period registers. The transfer functions for the output impedance and the audio susceptibility are denoted as $Z_{\text {out }}(s)$ and $G_{v g}(s)$.

In Fig. 5, it is easy to get the loop gains of the OVR loop by:

$$
T_{v o}(s)=3 K_{v o} G_{v d}(s) G_{v o}(s) / V_{D p p} .
$$

The IVS control loop gains can be expressed as:

$$
T_{v o}(s)=K_{v e r r c} G_{v e r r 1}(s) G_{\Delta v \Delta d 1}(s) / V_{D p p} .
$$

Based on the parameters for the ISOP configuration, the voltage sensor gains for the OVR and IVS loops are 0.1 and 0.006 respectively. $V_{D p p}$ is $6 \mathrm{~V}$. Both proportional-integral (PI) type compensations for output voltage and input voltage sharing control are made. During the uncompensated condition, the transfer functions of compensator $G_{v e r r 1}(s)$ and the compensator $G_{v 0}(s)$ equal 1 . The crossover frequency of the OVR loop is chosen to be $5 \mathrm{kHz}$ which is roughly one-seventh the switching frequency. In Fig. 6, the original loop gain of $G_{v d}(s)$ has a magnitude of $-30 \mathrm{~dB}$ at $5 \mathrm{kHz}$, but after compensation, the compensated loop gain has a crossover frequency of $5 \mathrm{kHz}$ with a phase margin of $40^{\circ}$. The bode diagram of the open loop transfer function $G_{\Delta v \Delta d}(s)$ is shown in Fig. 7, where the compensated loop gain has a crossover frequency of $3 \mathrm{kHz}$ with a phase margin of $30^{\circ}$.

\section{EXPERIMENTAL RESULTS}

Experiments on a prototype with the same parameters as those shown in the loop gain analysis have been made. It
TABLE I

SYSTEM SPECIFICATIONS

\begin{tabular}{|c|c|c|c|}
\hline Item & Value & Item & Value \\
\hline $\begin{array}{c}\text { Rated input } \\
\text { voltage } V_{i n}\end{array}$ & $800 \mathrm{~V}$ & Output capacitor $C_{f}$ & $1.0 \mathrm{~m} F$ \\
\hline Output voltage $v_{0}$ & $10 \mathrm{~V}$ & ESR of output capacitor $R_{c f}$ & $50 \mathrm{~m} \Omega$ \\
\hline Rated load $R_{0}$ & $1 \Omega$ & Output inductors $L_{f}$ & $0.1 \mathrm{~m} H$ \\
\hline $\begin{array}{c}\text { Switching } \\
\text { frequency } f_{s}\end{array}$ & $33 \mathrm{kHz}$ & $\begin{array}{c}\text { ESR of output } \\
\text { inductors } R_{L}\end{array}$ & $100 \mathrm{~m} \Omega$ \\
\hline
\end{tabular}

should be pointed out that the turns ratios of each module is not the same in fact. The turns ratios of transformer T1 and transformer T3 are both 4:1, while the turns ratio of T2 is 3:1. The digital control strategy for the proposed ISOP converter has been implemented by a DSP (TMS320F2812).

The TTF module contains the following power devices:

1) Switching devices: IXFH44N50P.

2) Diodes (clamping diodes, rectifier diodes and freewheeling diodes): DSEI30-06A.

Fig. 8 shows the gate signals for all of the modules with the proposed digital control strategy interleaved at full load, which can reduce the output current ripples. Because the turns ratio of $\mathrm{T} 2$ is less than those of the other two modules, the duty ratio for module 2 is smaller than those of module 1 and module 3 while the duty ratios of module 1 and module 3 are the same for identical turns ratios. Fig. 9 shows the primary voltages of the transformers in the system at full load under the steady state. Their amplitudes reveal that the corresponding individual input voltages are equal due to the excellent IVS control. Fig. 10 illustrates the individual converter input voltages corresponding to a step change in the input voltage from $660 \mathrm{~V}$ to $960 \mathrm{~V}$. As can be seen, before and after the transient, the input voltage can be shared equally among the three modules. Fig. 11 shows the individual converter input voltages corresponding to a load stepping between half load $(5 A)$ and full load $(10 A)$. Despite the transients, the total input voltage can be shared well too. Fig. 12 and Fig. 13 show the response of the individual input voltages and the output voltage during a load transient between full load and half load. As can be seen from the figures, with the proposed control strategy, the individual input voltages are almost unaffected when facing a load transient.

\section{CONCLUSIONS}

This paper proposes a digital control strategy for an ISOP converter consisting of double-switch forward type DC/DC converters, which can be used in high voltage input applications with low voltage rating switches. It is simple to generate interleaving PWM gate signals for the switches of each module, reducing the output current ripples as well as the size of output inductors. Small signal analysis of the ISOP converter has be made with the proposed digital control strategy. A $100 \mathrm{~W}$ three module prototype has been built to verify the effectiveness of the digital control strategy. Both under steady states and transients, the input voltage can be shared equally among all the modules despite turns ratios mismatches. 


\section{ACKNOWLEDGMENT}

This work was supported by the National Nature Science Foundation of China (Grant No.50807005).

\section{REFERENCES}

[1] F.H. Khan, et. al., "A multilevel modular capacitor - clamped DC-DC converter," IEEE Trans. Ind. Appl., Vol. 43, No. 6, pp. 1628-1638, Nov. 2007.

[2] J. W. Kim, et. al., "Modeling, control, and design of input-series-outputparallel-connected converter for high-speed-train power system," IEEE Trans. Ind. Electron., Vol. 48, No. 3, pp.536-544, Jun. 2001.

[3] R. Giri, V. Choudhary, R. Ayyanar and N. Mohan, "Common duty ratio control of input-series connected modular DC-DC converters with active input voltage and load-current sharing," IEEE Trans. Ind. Appl., 2006 Vol. 42, No. 4, pp. 1101-1111, Jul. 2006.

[4] P. J. Grbovic, "Master/slave control of input-series-and output-parallelconnected converters: concept for low-cost high-voltage auxiliary power supplies," IEEE Trans. Power. Electron., Vol. 24, No. 2, pp.316-328, Feb. 2009.

[5] X. B. Ruan, et. al., "Control strategy for input-series-output-parallel converters," IEEE Trans. Ind. Electron., Vol. 56, No. 4, pp. 1174-1185, Apr. 2009.

[6] S. P. Natarajan and T. S. Ananadhi, "Control of input series output parallel connected DC-DC converters," Journal of Power Electronics, Vol. 7, No. 3, pp.265-270, Jul. 2007.

[7] R.Ayyanar, et. al, "Active input-voltage and load-current sharing in input-series and output-parallel connected modular DC-DC converters using dynamic input-voltage reference sheme," IEEE Trans. Power. Electron., Vol. 19, No. 6, pp.1462-1473, Nov. 2004.

[8] R.Ayyanar, et. al,"Uniform voltage distribution control for series connected DC-DC converters," IEEE Trans. Power. Electron., Vol. 22, No. 4, pp.1269-1279, Jul. 2007.

[9] V.Choudhary, et. al "Fault tolerant circuit topology and control method for input-series and output-parallel modular DC-DC converters," IEEE Trans. Power. Electron., Vol. 23, No. 1, pp.402-411, Jan. 2008.

[10] J. P. Lee, B. D. Min,T. J. Kim, D .W. Yoo, and J.Y.Yoo, "Input-seriesoutput-parallel connected DC/DC converter for a photovolatic PCS with high efficieency under a wide load range," Journal of Power Electronics, Vol. 10, No.1, pp.9-13, Jan. 2010.

[11] D.V.Ghodke, et. al, "ZVZCS, dual,two-transistor forward DC-DC converter with peak voltage of Vin/2,high input and high power applications," in Proceeding of IEEE_PESC, Cairns, pp. 1853-1858, Jun. 2002.
[12] D. S. Sha, et. al "Digital control of switch-mode pulsed welding power," in Proceeding of IEEE_ECCE, San Joes, pp. 2746-2749, Sep. 2009.

[13] S.Bibian, et. al, "High performance predictive dead-beat digital controller for DC power supplies," IEEE Trans. Power. Electron., Vol. 17, No. 3, pp.420-427, May 2002.

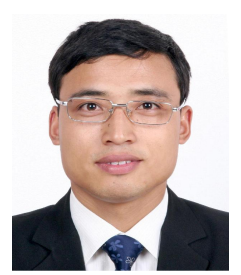

Deshang Sha was born in 1977. He received his B.S from Luoyang Institute of Technology, Luoyang, China, in 1998, his M.S. from the Nanjing University of Aeronautics and Astronautics, Nanjing, China in 2001 and his $\mathrm{Ph} . \mathrm{D}$. from the Institute of Electrical Engineering, Chinese Academy of Sciences, Beijing, China in 2005, all in Electrical Engineering. From 2005 to 2007, he worked as the Head and Chief Engineer of the fulldigitalized welding machine research team of Time Group Company. Since 2008, he has been with the School of Automation, Beijing Institute of Technology, where he is currently a Lecturer. His research interests are in the areas of digital control of power electronics and renewable energy power generation systems.

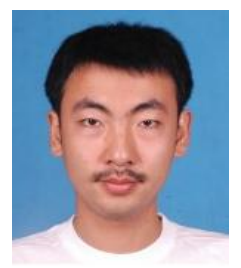

Zhiqiang Guo was born in 1985. He received his B.S in Automation from the Hebei University of Technology, Tianjin, China, in 2008. He is now pursing his Ph.D. in Electrical Automation at the Beijing Institute of Technology. His research interests are in the areas of modeling and control of power electronics.

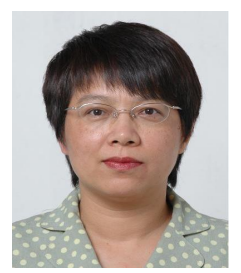

Xiaozhong Liao was born in China, in 1962. She received her B.S. and M.S. in Electrical Engineering from Tianjin University, Tianjin, China, in 1982 and 1984, respectively, and her Ph.D. in Control Sciences and Engineering from the Beijing Institute of Technology, Beijing China, in 1994. She was a Visitor Researcher in the Department of Electrical and Electronic Engineering, University of Central Lancashire, Preston, U.K. form 1995 to 1996. She is now an Associate Dean and Professor in the School of Automation, Beijing Institute of Technology. Her research interests are in the fields of power electronics, motor drives and renewable energy power conversion. 